\title{
Fast improvised diagnostic robust measure for the identification of high leverage points in multiple linear regression
}

\begin{abstract}
High leverage points in the data set have massive effect in linear regression. Identifications of high leverage points (HLP) is very important due to their effect of causing wrong conclusion in regression model. The Diagnostic Robust Generalized Potential based on Index Set Equality IDRGP (ISE) is very successful in identifying high leverage points with less running time. However, it still reveals smaller rate of masking and swamping. A fast Improvised method for the identification of High Leverage Point is proposed to reduce the effect of swamping and masking at a faster rate of running time. The results of the simulation study show the merit of our proposed method.
\end{abstract}

Keywords: Masking; Swamping; Index Set Equality; Improvised Diagnostic Robust Generalized Potential 